\title{
Results and lessons learnt from a randomized controlled trial: prophylactic treatment of vestibular migraine with metoprolol (PROVEMIG)
}

Otmar Bayer ${ }^{1,2+}$, Christine Adrion ${ }^{3 *+} \mathbb{D}$, Amani Al Tawil ${ }^{3}$, Ulrich Mansmann ${ }^{3}$, Michael Strupp ${ }^{1,4}$ and PROVEMIG investigators

\begin{abstract}
Background: Vestibular migraine (VM) is the most frequent cause of recurrent spontaneous attacks of vertigo causally related to migraine. The objective of the Prophylactic treatment of vestibular migraine with metoprolol (PROVEMIG) trial was to demonstrate that metoprolol succinate is superior to placebo in the prevention of episodic vertigo- and migraine-related symptoms in patients with VM.

Methods: This phase III, two-arm, parallel-group, double-blind, randomized placebo-controlled trial was designed to be conducted at tertiary referral centres at neurology and ear, nose and throat departments of eight German university hospitals. The planned sample size was a total of 266 patients to be allocated. Adults aged 18 years or above diagnosed with probable or definitive VM according to the Neuhauser criteria 2001 were randomly assigned 1:1 to 6 months blinded metoprolol (maintenance dosage of $95 \mathrm{mg}$ daily) or placebo. The primary efficacy outcome was the self-reported number of vertiginous attacks per 30 days documented by means of a paper-based daily symptom diary. The pre-specified time period of primary interest was defined as months 4 to 6 . Secondary outcomes included the patient-reported number of migraine days and vertigo days, the Dizziness Handicap Inventory, and clinical assessments. Adverse events were reported throughout the whole 9-month study period.

Results: At the time of trial termination, no evidence for a difference in the incidence of vertiginous attacks between groups was detected. For the full analysis set, the incidence rate ratio was 0.983 ( $95 \%$ confidence interval (Cl) 0.902-1.071) for metoprolol versus placebo. In both groups, there was a significant decline over time in the overall monthly vertigo attacks by a factor of 0.830 ( $95 \%$ Cl $0.776-0.887$ ). Results were consistent for all subjective and objective key measures of efficacy. The treatment was well tolerated with no unexpected safety findings.

\footnotetext{
* Correspondence: adrion@ibe.med.uni-muenchen.de

+Otmar Bayer and Christine Adrion contributed equally to the work and share first authorship.

${ }^{3}$ Institute for Medical Information Processing, Biometry and Epidemiology (IBE), Ludwig Maximilians University, Campus Grosshadern, Marchioninistr. 15, 81377 Munich, Germany

Full list of author information is available at the end of the article
}

(c) The Author(s). 2019 Open Access This article is distributed under the terms of the Creative Commons Attribution 4.0 International License (http://creativecommons.org/licenses/by/4.0/), which permits unrestricted use, distribution, and reproduction in any medium, provided you give appropriate credit to the original author(s) and the source, provide a link to the Creative Commons license, and indicate if changes were made. The Creative Commons Public Domain Dedication waiver (http://creativecommons.org/publicdomain/zero/1.0/) applies to the data made available in this article, unless otherwise stated. 


\begin{abstract}
(Continued from previous page)
Conclusions: After randomizing 130 patients PROVEMIG had to be discontinued because of poor participant accrual not related to the tolerability of the study medication or safety concerns; no treatment benefit of metoprolol over placebo could be established. Additional preparatory work is much needed in the development, psychometric evaluation and interpretation of clinically meaningful end points in trials on episodic syndromes like VM taking into consideration the complexity of this disease entity comprising two domains (vertigo- and headacherelated disability).
\end{abstract}

Trial registration: EudraCT, 2009-013701-34. Prospectively registered on 8 April 2011.

Keywords: Vestibular migraine, Episodic migraine, Patient-centred outcomes, Symptom diary, Pharmaceutical intervention, Pharmacological prophylaxis, Randomized controlled trial, Comparative effectiveness

\begin{abstract}
Lessons learnt
- Thus far, there are no definitive specific curative or preventative therapies available for vestibular migraine (VM).

- This is the first pragmatic phase III, double-blind, randomized placebocontrolled superiority trial in adults with definite or probable VM comparing metoprolol as a prophylactic medication against placebo. - There are important implications for the planning stage of future randomized controlled trials in VM with respect to placebo or nonspecific and drug-specific effects.

- The PROVEMIG trial was prematurely ended due to insufficient recruitment. Reasons for the poor participant accrual were multifactorial and included lowered patient acceptability, unwillingness to accept the underlying intervention (being on antihypertensive therapy),

comorbidities being contraindications for metoprolol, or uncertainties in diagnosis.

- For VM, there is a strong need to develop and validate clinically meaningful, consensus-based patient-centred core outcome measures considering both the vestibular- and headache-related disease burden and to assess their psychometric performance.
\end{abstract}

\section{Background}

During the past decades, vestibular migraine (VM) has been identified as a type of migraine with the leading symptom of vertigo. Recently, it has been accepted as a distinct diagnostic entity by the Bárány Society and the International Headache Society [1]. Since vertigo frequently occurs in isolation and is not always accompanied by a headache or other migrainous symptoms, there is a strong need for accepted diagnostic criteria; these were first published in 2001 by Neuhauser and colleagues [2] and later refined by the International Classification of Vestibular Disorders of the Bárány Society [3].

Based on validated neuro-otologic interviews [4, 5], the prevalence of migrainous vertigo in the general adult population was estimated in a large German neurootologic survey; its lifetime prevalence was $0.98 \%$ and the 12 -month prevalence $0.89 \%$ [6]. The similarity of these two numbers suggests that these patients suffer chronically from this condition. A more recent survey from the USA found a prevalence of $2.7 \%$ in adults [7]. In a specialized dizziness clinic, VM is the most frequent cause of spontaneous recurrent attacks of vertigo and accounts for approximately $10 \%$ of the patients [8]. The majority of patients with VM are middle-aged and in the middle of their working lives.

With a lack of double-blind, randomized controlled clinical trials [9], recommendations for treatment are aligned to those of migraine $[10,11]$. The following drugs have been recommended as prophylactic treatment for VM: beta blockers, valproic acid, lamotrigine [12], tricyclic antidepressants and topiramate [13]. In an observational study on 81 patients, the effects of tricyclic antidepressants, beta blockers, or calcium-channel blockers in combination with diet were evaluated. Seventy-two percent of the patients showed a good response [14]. More recently, flunarizine [15], propranolol and venlafaxine [16] have been investigated in activecontrolled, open-label trials. Metoprolol is listed as a group 1 medication (drug of first choice) in a dose of 50 to $200 \mathrm{mg}$ and has shown efficacy in prophylactic treatment of migraine $[17,18]$. Due to the absence of consensus guidelines for the treatment of VM, beta blockers such as metoprolol are commonly prescribed as off-label preventive pharmacologic treatment in VM.

For this reason, the Prophylactic treatment of vestibular migraine with metoprolol (PROVEMIG) trial was conducted. This investigator-initiated, prospective, longitudinal, national, multicentre, double-blind, randomised, placebo-controlled, two-arm parallel group, phase III, pragmatic superiority trial aimed to evaluate the effectiveness, safety and tolerability of metoprolol succinate versus placebo for the preventive treatment of VM. Treatment duration in both arms was 6 months, with a 3 -month post-treatment follow-up period. The primary objective was to demonstrate the superiority of metoprolol with respect to the incidence rate of vertiginous attacks. Further secondary objectives included comparing both regimens with respect to vertigo and headache characteristics, to investigate changes in neurological and neuro-ophthalmological assessments, and vertigorelated impairment of quality of life, and to further establish the safety profile of the drug. We report the prespecified efficacy and safety analyses for the 6-month 
treatment period following reporting guidelines for trials describing patient-reported outcomes and related extensions (the checklist for the CONSORT 2010 statement is provided as Additional file 1) [19-21].

\section{Methods}

\section{Study oversight}

The study was investigator-initiated and conducted in accordance with the principles of the Declaration of Helsinki, the International Conference for Harmonisation Guidelines for Good Clinical Practice, and relevant national regulations. The protocol (Additional file 2) was approved by the ethics committees of each participating centre. Furthermore, the efficacy, safety, integrity and feasibility of the trial were monitored by a Data Safety Monitoring Board consisting of three independent, nonparticipating clinicians. All patients provided written informed consent before any study procedures or assessments were performed.

\section{Study population and procedures}

Subjects were screened at six German academic outpatient clinics; four of these investigational sites (German Center for Vertigo and Balance Disorders at the University Hospital Munich; Department of Neurology of the General Hospital Celle; the University of Essen; and the community hospital at AltöttingBurghausen) allocated patients between 20 June 2012 (first patient) and 10 April 2017; the last patient visit was on 3 January 2018.

The patient population consisted of male or female patients aged 18 years and above diagnosed as having probable or definite VM according to the Neuhauser criteria [2] (see Additional file 2 for details). For enrolment, patients had to experience a frequency of 6 to $30 \mathrm{VM}$-related attacks per 3 subsequent months prior to the screening visit (information retrospectively collected at in-person interviews), be capable of following the study instructions and be likely to complete the study visits.

Exclusion criteria were a diagnosis of other co-existing vestibular disorders such as Menière's disease, phobic postural vertigo, benign paroxysmal positional vertigo (BPPV) and vestibular paroxysmia. Patients were also excluded if they had central disorders such as paroxysmal brainstem attacks and transient ischemic attacks. Other exclusion criteria were contraindications for the treatment with metoprolol such as a known allergic reaction to the trial drug or other beta-receptor blockers, shock, acidosis, any bronchospastic disease (e.g. bronchial asthma), sick sinus syndrome, known sino-atrial or atrio-ventricular block, bradycardia of less than $50 \mathrm{bpm}$ at rest, systolic blood pressure less than $100 \mathrm{mmHg}$, end-grade peripheral arterial disease, known severe coronary heart disease or heart failure and concurrent treatment with monoamine oxidase inhibitors, sympathomimetic drugs, catecholamine-depleting drugs, or digitalis glycosides. Other patient factors leading to exclusion included poorly controlled diabetes mellitus, pheochromocytoma, suspicion of developing thyrotoxicosis, disorders of homeostasis, porphyria, psoriasis, pregnancy or breastfeeding, persistent hypertension with systolic blood pressure higher than $180 \mathrm{mmHg}$ or diastolic blood pressure higher than $110 \mathrm{mmHg}$ (mean of three consecutive arm-cuff readings over 20 to $30 \mathrm{~min}$ ) that cannot be controlled by antihypertensive therapy, life expectancy of less than 12 months, other serious illness that may confound treatment assessment, currently receiving beta blockers, enrolment in another clinical trial, and exposure to any investigational medication within 30 days prior to the baseline visit.

\section{Study procedures}

This study consisted of a screening visit, a 6-month treatment period, and a final evaluation at month 9 after a 3-month post-treatment follow-up period. Both the study examinations and treatment were performed in an outpatient setting. Based on the information from the screening visit, patients were either randomized or were excluded if they did not meet eligibility criteria. On the day of inclusion, patients received their study medication together with a paper-based diary to document VMrelated symptoms on a daily basis over the 9-month observation period. Patients were seen at five scheduled clinic visits for protocol-specified evaluations at screening, baseline (day of inclusion), and at months 1,3 , and 6 (end of treatment period); three standardized telephone interviews were performed after 2, 4, and 5 months post-randomization for compliance checks with respect to treatment and diary documentation, and safety assessment.

All enrolled patients underwent a physical examination at the baseline visit, and at every clinic visit post-randomization non-invasive neurological and neuro-otological and neuro-ophthalmological examinations such as video-oculography including bithermal caloric testing, assessment of pursuit eye movement, gazeholding, saccades and subjective visual vertical (SVV) were performed. For more details refer to the original protocol in Additional files 2 and 4. Furthermore, participants had to complete the paper-based, self-administered Dizziness Handicap Inventory (DHI), a 25 -item validated questionnaire with a 3-point response scale to rate the self-perceived impact of dizziness on health-related quality of life [22, 23]. Possible DHI total scores range from 0 to 100 points, with higher values reflecting greater impairment. Results from the trial assessments were recorded in 
paper-based case report forms filled in by the study personnel at each clinical site.

\section{Event-driven diary documentation}

Dizziness event data were captured by means of the patient's diary with entries made whenever symptoms associated with migrainous vertigo occurred. Patients were instructed to document the following items: time of onset, duration, severity and type of the vertigo symptom (rotatory or postural vertigo, gait unsteadiness, or light-headedness); occurrence of accompanying symptoms (headache, nausea, vomiting, photo- or phonophobia, diplopia, other visual symptoms, tendency to fall); any action taken including any medication use. A diary template (original German version together with an English translation) is provided in Additional file 3.

\section{Randomization, concealment and blinding}

Patients who met the eligibility criteria for enrolment were randomized in a 1:1 ratio to receive either metoprolol succinate or placebo for 6 months (Fig. 1). Each study site received a pool of study medication including the treatment assignment in an opaque, sealed emergency envelope. If an eligible patient dropped out before the study medication had been delivered they were replaced by the next eligible patient enrolled at the same site. The concealed allocation was performed by an internet-based randomization schedule stratified by study site (https://wwwapp.ibe.med.uni-muenchen.de/ randoulette). The fixed block size was four (starting with 6) which was not disclosed during the trial. The random number list was generated by a person with no clinical involvement in the trial. Patients and site personnel including outcome assessors, data analysts and statisticians remained blinded to treatment allocation.

\section{Study treatments}

Metoprolol succinate sustained-release tablets (Beloc-Zok ${ }^{\circ}$ mite $47.5 \mathrm{mg}$; manufactured by AstraZeneca, Wedel, Germany) were encapsulated for blinding purposes. Hard gelatine capsules containing the active ingredient were refilled from original pharmacy packaging into re-labelled blisters by the pharmacy of the University Hospital in Heidelberg, Germany. Placebo was an identically appearing inactive capsule filled with mannitol and aerosil that did not contain any active ingredient; this was packed in blisters that looked identical to those of the investigational drug. Randomized patients were instructed to take one capsule per day starting as soon as possible after the receipt of the trial medication kit dispensed at the baseline visit. The treatment procedure included a 1-week run-in period of $47.5 \mathrm{mg}$ metoprolol succinate or placebo once a day (up-titration), a 6-month maintenance dosage with 95 mg metoprolol succinate or placebo once a day, plus tapering with $47.5 \mathrm{mg}$ metoprolol succinate or placebo once a day for 2 weeks before stopping the prophylactic therapy (down-titration). Placebo treatment was justified due to a lack of well-designed placebo-controlled trials for any drug therapy in VM. The 6-month treatment duration was deemed necessary to reliably assess a long-term prophylactic effect of the drug treatment on the incidence of VM-related vertigo attacks. If the patient was on prophylactic drug treatment for migraine, a washout period of at least 1 month was required before enrolment. Topiramate, valproic acid, lamotrigine, tricyclic antidepressants and other beta blockers were considered as prohibited concomitant medication and thus a protocol violation. Acute medical treatment of VM-related attacks such as episodic migraine with aura using non-opioid analgesics, non-steroidal anti-inflammatory drugs or triptans was allowed, serving as added rescue medication. We aimed to assess the comparative effectiveness of the assigned prophylactic treatment regardless of whether or not switching to rescue medication had occurred which can be denoted as 'treatment policy estimand' according to the International Council for Harmonisation E9 addendum [24].

\section{Statistical methodology and planned analyses Protocol-defined efficacy outcomes and changes after trial commencement}

The primary objective was to assess whether metoprolol was superior to placebo with respect to both disease domains of 'vertigo' and 'headache'. For the purpose of the study, the target estimate was based on the overall monthly mean incidence of vertigo and headache attacks during a 3-month-long assessment period at the end of the double-blind, 6-month treatment period, i.e. months 4 to 6 (day 91 to day 180) was defined as the time period of primary interest assuming that the maximum treatment effect emerges after being on study medication for more than 3 months. The pre-specified primary efficacy outcomes were the patient-reported number of vertigo attacks and the number of headache attacks per 30-day interval (starting from time point 1 defined as the date of first intake of the study medication). According to the protocol, superiority was to be claimed based on the vertigo outcome domain alone. Thus, the incidence of headache attacks per 30 days was defined as a coprimary outcome. In case of claimed superiority with respect to the outcome domain 'vertigo', the comparison of the monthly incidence of headache attacks between both groups was to be considered next important.

However, due to the poor documentation concerning headache-related symptoms and the diary focussing on the vestibular symptoms, derivation of a measurable variable for headache attacks was considered impossible and the co-primary efficacy end point had to be omitted. 


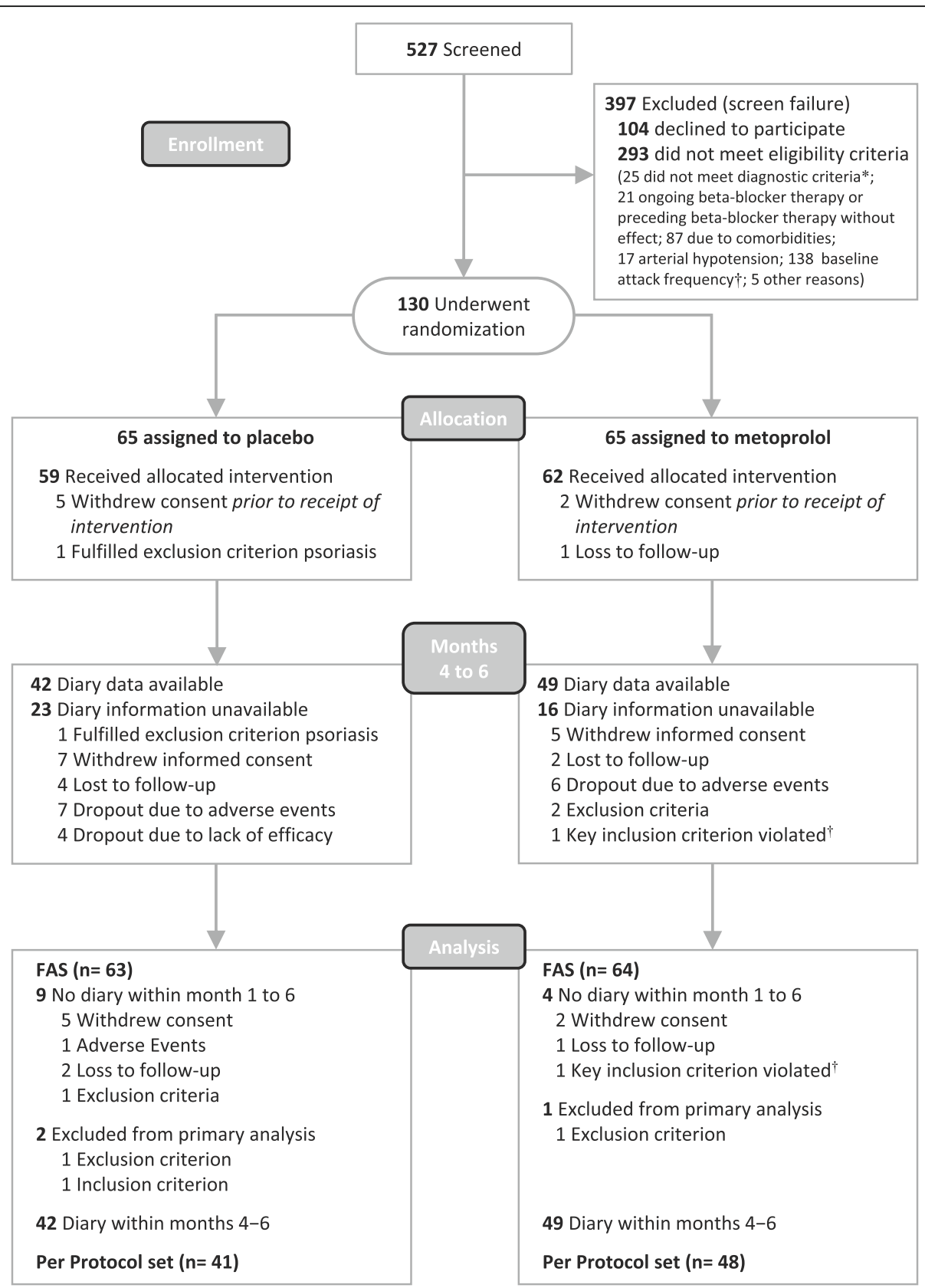

Fig. 1 Consolidated Standards of Reporting Trials (CONSORT) 2010 flow diagram together with patient-reported outcome specific information. Enrolment and primary efficacy end points based on patient diaries. The steps lead from pre-screening to collection of the data used in the efficacy analyses. The diagram shows the extent of exclusions, loss to follow-up and missing data within the 6-month treatment period (diary information unavailable means no diary at all within the time period of primary interest, day 91 to day 180). *Diagnostic criteria according to Neuhauser 2001 [2]. 'Baseline frequency of vertigo attacks in the last 3 subsequent months prior to enrolment. Per protocol: treatment duration $>90$ days, counting from day of first intake. FAS full analysis set

Furthermore, the initially planned secondary outcomes duration and severity of vertigo episodes were omitted due to insufficient data quality. Instead, the number of vertigo days per 30 days (which was not pre-registered in the protocol) was defined as a clinically meaningful key secondary efficacy outcome to assess the disease burden with respect to the outcome domain 'vertigo'. A vertigo day was defined as a calendar day (00:00 to 23:59) demonstrating at least one documented vertigo episode of at least $5 \mathrm{~min}$ (regardless of severity and type). Derivation of this efficacy variable relies on fewer assumptions compared to a vertigo attack and also enables handling missing diary items.

On the contrary, a vertigo attack was endorsed applying the following decision rules: duration of at least $5 \mathrm{~min}$ and 
no longer than $72 \mathrm{~h}$, irrespective of vertigo type and severity; if time data (start and stop time) for a vestibular symptom were absent a duration of $24 \mathrm{~h}$ was assumed; a vertigo episode which was interrupted by sleep or temporarily remits was classified as one single attack, and not two; if applicable, patient-reported vertigo symptoms reported on 2 or more consecutive calendar days were summarized to one single vertigo episode lasting over consecutive calendar days (however, if the resulting duration extends beyond $72 \mathrm{~h}$ these calendar days were considered free of vertigo attacks but counted as vertigo days).

A pre-specified diary-based secondary efficacy outcome was the number of monthly headache days (a calendar day where headache of any severity occurred according to the patient ratings). During the blind data review, this patient-reported outcome was refined to as a migraine headache day (MHD), requiring at least one additional migraine-associated symptom such as nausea, vomiting, phono- or photophobia, disturbance of vision, or "migraine" provided as free text on the diary. However, features such as duration and severity of migraine headache or criteria as proposed by the International Classification of Headache Disorders-3 from 2018 [1] were not considered in order to derive definite MHD since these items were not requested on the diary.

All these changes to the latest protocol version concerning efficacy evaluation were made before breaking the treatment blind thus minimizing outcome reporting bias. Owing to the complexity of vestibular and migraineassociated symptoms, inaccurately documented episodes of vertigo (e.g. missing outcome items), and different individual perceptibility of both domains of the disease, the assessment of vertigo attacks, days and MHDs based on the raw daily diary recordings was very challenging. Therefore, a computer algorithm programmed in SAS was developed for the process of outcome adjudication and to derive all diary-based efficacy variables.

Protocol-defined observer-reported secondary efficacy end points included the proportion of patients achieving an improvement from baseline to month 6 in pursuit eye movement (change from 'saccadic (of any direction)' to 'smooth' versus change from 'smooth' to 'saccadic', or no change), and the proportion of patients achieving an improvement in SVV (change from 'abnormal' to 'normal' versus change from 'normal' to 'abnormal' or no change; 'abnormal' was defined as the absolute deviation of more than $2.5^{\circ}$ from vertical). Furthermore, the absolute change in the DHI mean total score from baseline to month 6 was assessed.

\section{Statistical efficacy analyses (including changes to protocol- specified analyses)}

Efficacy analyses were conducted for the full analysis set (FAS) which included all randomized patients (intentionto-treat population) who did not fail to satisfy a major entry criterion, irrespective of whether they were treated or not. Subjects who provided neither primary nor secondary efficacy data were excluded from efficacy analyses, assumed missing at random (MAR). The perprotocol sample consisted of all participants who were part of the FAS who did not substantially deviate from the protocol and who were on treatment for more than 90 days, counting from the day of first intake. Safety analyses were performed on all patients who received at least one dose of study drug.

According to the protocol, the principal analysis for the primary end point incidence of vertigo attack per 30 days during the 3-month time period of primary interest (months 4 to 6) was a robust non-parametric comparison between both treatment groups by means of the Wilcoxon rank-sum test. However, in the course of the trial it was evident that drop-outs and incomplete diary documentation creating missing data could not be adequately handled by the intended test-based approach. In order to deal with the missing data structure over time we used a Poisson mixed effects model (Poi generalized linear mixed model) which not only yields unbiased parameter estimates when missing observations are MAR, but also provides reasonably stable results even when the assumption of MAR is violated [25-27].

For this longitudinal model-based approach, the logtransformed number of evaluated days per 30 days (defined as the number of calendar days with assessments recorded in the diary within a 30-day interval) was considered as an offset term in order to reflect missing diary information (e.g. for withdrawals) and to standardize the monthly incidence of vertigo attacks to 30 days for that month. Time (range 1 to 6 ) and treatment-by-lineartime interaction were used as fixed effects, together with patient-specific intercepts and slopes for time as normally distributed random effects. Assuming no rapid onset of effect staying stable over time, the main fixed effect for the treatment group was omitted. The target estimates consist of the decay rate for the placebo group (fixed effect for time) as well as the incidence rate ratios (IRRs) for the metoprolol group (treatment-by-time interaction) to assess if the magnitude of the difference between treatment groups varies over time. The latter can be interpreted as 'speed of efficacy' [28]; that is, whether the active agent may be distinguished from placebo by how quickly a reduction in the incidence of attacks was achieved. The same longitudinal model approach was applied for vertigo days serving as a supplementary analysis.

MHDs per 30 days were analysed with a negative binomial model (with an offset term for the corresponding number of evaluated days during the 90day assessment period) using self-reported symptoms documented within months 4 to 6 only. An analysis of 
covariance for absolute change from baseline in DHI mean total score at month 6 was performed, which used a factor for treatment group and the baseline value as a covariate.

For the binary response measures of change in state from baseline in SVV and pursuit eye movement at month 6, a logistic regression analysis was conducted (1, from abnormal to normal; 0, otherwise).

\section{Safety and tolerability}

Adverse events (AEs) and tolerability were systematically assessed by evaluating reported AEs, physical examinations and concomitant medication use. The safety population included data from all randomized patients who received at least one dose of the investigational medicinal product during the double-blind treatment phase. Serious AEs (SAEs) were coded and summarized by the Medical Dictionary for Regulatory Activities (MedDRA) system organ class and preferred term. For some AEs, the exact starting date (day and/ or month) was partially or completely missing. In order to deal with the different input accuracy or partial date issues, AEs were classified with respect to their occurrence (AEs emerging while on treatment versus post-treatment AEs) assuming the $\mathrm{AE}$ was experienced at the earliest possible date.

\section{Sample size considerations}

A fixed sample size calculation was performed for the primary efficacy outcome (number of vertigo attacks). A sample size of 106 patients in each group will have 80\% power to detect a probability of 0.389 that an observation $X_{M}$ is less than an observation $X_{P}$ using a Mann-Whitney test with a $5 \%$ two-sided significance level. The probability of $P\left(\mathrm{X}_{\mathrm{M}}<\mathrm{X}_{\mathrm{P}}\right)=0.611$ was calculated with a presumed normal distribution and difference in means of 1 and a standard deviation of 2.5 (nQuery Advisor 7.0). On the basis of our experience with patient compliance in previous studies and routine treatment, we assumed a drop-out rate of about $20 \%$. Thus, the fixed target sample size was a total of 266 patients (133 in each treatment group) to be allocated. Further detailed descriptions on how the sample size was calculated are provided in Additional file 2.

The study database was stored in SAS (Unix Version 9.2, SAS Institute, Cary, NC, USA). Statistical analyses were performed using the statistical software package $\mathrm{R}$ version 3.5.1 [29]. For the efficacy analyses we used the $\mathrm{R}$ package lme4 (version lme4_1.1-18-1) to fit frequentist generalized linear mixed effects models [30, 31]. All statistical tests were two-sided, with a significance level of 0.05 .

\section{Results}

Premature termination of the trial

In June 2017, the sponsor-delegated person together with the responsible biometrician and the Data Safety
Monitoring Board prompted an early termination of the study on the grounds of poor patient accrual after randomization of 130 patients, and not for any reasons related to safety.

Financial resources for the continuation of the PROVEMIG trial were no longer available due to lack of funding. To reach the a priori determined target sample size of 266 patients in total, further years and more recruiting sites would have been required, which was considered not feasible. Further concerns were the fact that the monthly recruitment rates in the participating sites were lower than anticipated and decreasing over time (Additional file 4: Figure S1). Overall, early stopping of the trial for feasibility reasons at the risk of generating an underpowered trial providing inconclusive data seemed justified.

\section{Patient disposition and baseline characteristics}

At the time of the study termination, 527 patients had been screened for eligibility at six sites. Despite constant attempts to increase recruitment rates at the participating sites, randomization was stopped after 130 patients were enrolled, 109 (84\%) of them at the sponsor-delegated person's site in Munich. The most common reasons for screening failure were failure to meet criteria for enrolment such as low baseline severity level with respect to VM-related attack incidence prior to enrolment, refusal to provide informed consent, excluded comorbidities, and ongoing beta-blocker therapy due to indications not necessarily associated with VM.

Figure 1 illustrates the flow of patients through the trial together with patient-reported outcome specific information [20]. In total, 130 patients were allocated to either metoprolol or placebo and were included in the intention-to-treat population. The FAS population consisted of 127 patients with three patients being excluded after randomization: two patients allocated to placebo (concomitant diagnosis of BPPV causing inability to discriminate episodes caused by VM from the ones caused by BPPV) did not fulfil the major entry criterion with respect to baseline attack severity; and one patient allocated to metoprolol fulfilled a major exclusion criterion (concomitant diagnosis vestibular paroxysmia with no diary information provided). In the placebo group, nine patients did not provide any diary information compared to four patients in the metoprolol group. Within the 3month assessment period, diary data were available for 91 out of 127 patients of the FAS sample (42 patients in the placebo group versus 49 patients in the metoprolol group). The proportion of intermittent missing information was rather low for both treatment groups whereas the proportion of monotone missing diary information, e.g. due to treatment adherence or noncompliance, was rather high. Besides, the proportion of missing diary data 
was higher in the placebo than in the metoprolol group (Additional file 4: Figure S2).

Table 1 gives the demographic and important clinical characteristics including the vertigo-specific quality-oflife DHI score of all randomized patients assessed at the baseline visit. Overall, $60.8 \%$ of the randomized patients were female and the median age was 44 years. The proportion of patients diagnosed with definite (as opposed to probable) VM was $61.5 \%$, with $64.6 \%$ in the metoprolol group being slightly higher than $58.5 \%$ in the placebo group. Overall, both groups were similar to each other in respect of demographics and baseline clinical patient characteristics. Pre-randomization attack frequency with respect to the domains of vertigo and migraine was not documented although considered as a key inclusion criterion. No information with regard to disease duration or age at onset was available.

\section{Dosing and adherence to initial treatments}

In total, 121 patients received at least one dose of the study medication. In the FAS, the median treatment duration (range) was 177 (0-203) days in the placebo group, and 178 (0-236) days in the metoprolol group (the difference in location of median duration $(95 \%$ confidence interval (CI)) was -1.999 (-6.000 to 2.999; $P=0.451$ ). A considerable number of patients took rescue medication on an as-needed basis. However, the proportion was comparable in both randomized groups (data not shown).

\section{Primary and key secondary efficacy analyses}

For the FAS sample, 114 patients (54 on placebo, 60 on metoprolol) contributed data to the Poisson mixedeffects model which revealed an overall relief in vertigorelated symptoms over time in both treatment groups. The mean incidence rate of vertigo attacks in those receiving placebo was significantly reduced by a factor of 0.830 per additional month while on treatment $(95 \% \mathrm{CI}$ $0.776-0.887 ; P<0.001)$. It was hypothesized that the assigned active treatment would make this overall decay rate even smaller. The corresponding estimated factor, representing the IRR compared to placebo, was 0.983 (95\% CI 0.902-1.071) in those receiving metoprolol; no evidence for a treatment-by-time interaction was found (global testing, likelihood-ratio test, $P=0.696$ ) indicating no statistically significant difference in the monthly incidence rates for vertigo attacks between both groups. Table 2 displays the results for months 4,5 and 6, representing the pre-specified 3-month time period of primary interest to assess treatment effectiveness. Within month 6 , the marginal mean incidence rate per 30 days for vertigo attacks was 3.097 (95\% CI 1.914-4.281) for patients receiving placebo versus 2.796 (95\% CI 1.7923.800) for those receiving metoprolol. Similarly, for the per-protocol sample (comprising of 89 patients; 41 receiving placebo), the overall decay rate was 0.848 (95\% CI $0.795-0.904 ; P<0.001)$ and the IRR was estimated to be 0.978 (95\% CI $0.901-1.061 ; P=0.593)$ indicating a lack of a beneficial treatment effect.

Considering the robustness of the primary result, a supplementary analysis for vertigo days was conducted in line with the primary efficacy analysis. For the FAS sample, the mean monthly incidence of vertigo days was reduced by $13 \%$ per additional month on placebo treatment, i.e. by a factor of 0.870 (95\% CI $0.821-0.923$; $P<0.001$ ). However, no superiority of metoprolol compared to placebo was found, with an estimated IRR of 0.940 ( $95 \%$ CI 0.869 to $1.017 ; P=0.125$ ). Figure $2 \mathrm{a}$ depicts the estimated monthly incidence rates for vertigo attacks during the whole 6-month treatment period for the placebo and metoprolol group. Figure $2 \mathrm{~b}$ shows the estimated monthly incidence rates over time for the key secondary outcome of vertigo days. During the 3-month assessment period (months 4 to 6 ) the mean monthly incidence for MHD was similar in the placebo and metoprolol group. The corresponding IRR defining the treatment effect estimate was 1.048 (95\% CI $0.482-$ 2.250; $P=0.904$ ).

Table 3 summarizes the results for planned key secondary efficacy outcome analyses at the pre-specified time point of month 6 . The DHI mean total score evaluating the self-perceived handicapping effects imposed by VM remained fairly stable at the end of the treatment period compared to the score measured at the baseline visit. The complete case analysis of covariance revealed no evidence for a between-treatment difference in mean change scores $(\Delta=-0.079$ ( $95 \%$ CI -0.360 to $0.201 ; P=$ $0.577)$ ). No statistically significant and clinically meaningful difference between placebo and metoprolol could be detected for smooth pursuit eye movement and SVV assessments. For both clinician-reported end points, the chance of achieving treatment response, i.e. a change from 'abnormal' at baseline to 'normal' at month 6, did not differ between both groups. For smooth pursuit eye movement, the odds ratio (OR) was estimated to be 1.483 (95\% CI $0.454-5.277 ; P=0.520$ ), and, for SVV, the OR was $0.413(95 \%$ CI $0.055-2.235 ; P=0.322)$ for metoprolol compared with placebo. Hence, patients assigned to metoprolol did not achieve superior patient-reported and clinician-reported outcomes compared to those assigned to placebo treatment, suggesting robustness of the principal result.

\section{Safety and tolerability}

Since metoprolol succinate is a well-established drug that has been used for many years in common diagnoses such as hypertension and episodic or chronic migraine, it was expected to be generally well tolerated here. 
Table 1 Baseline and disease characteristics of the intention-to-treat sample

\begin{tabular}{|c|c|c|}
\hline Characteristics & $\begin{array}{l}\text { Placebo } \\
(n=65)\end{array}$ & $\begin{array}{l}\text { Metoprolol } \\
(n=65)\end{array}$ \\
\hline \multicolumn{3}{|l|}{ Demographics } \\
\hline \multicolumn{3}{|l|}{ Age (years) } \\
\hline Mean (SD) & $42.8(14.3)$ & $44.4(14.2)$ \\
\hline Median (range) & $44.0(19-70)$ & $45.0(19-75)$ \\
\hline \multicolumn{3}{|l|}{ Male sex } \\
\hline$n(\%)$ & $29(44.6)$ & $22(33.8)$ \\
\hline \multicolumn{3}{|l|}{ VM diagnostic criteria } \\
\hline Probable VM, n (\%) & $27(41.5)$ & $23(35.4)$ \\
\hline Definite VM, $n(\%)$ & $38(58.5)$ & $42(64.6)$ \\
\hline \multicolumn{3}{|l|}{ General physical examination } \\
\hline \multicolumn{3}{|l|}{ Body mass index $\left(\mathrm{kg} / \mathrm{m}^{2}\right)$} \\
\hline Mean (SD) & $26.4(5.5)$ & $25.9(3.9)$ \\
\hline Median (range) & $25.3(17.6-46.6)$ & $26.0(17.5-38.1)$ \\
\hline Missing, $n(\%)$ & $5(7.7)$ & $6(9.2)$ \\
\hline \multicolumn{3}{|l|}{ Systolic blood pressure (mmHg) } \\
\hline Mean (SD) & $134.1(18.6)$ & $136.8(16.3)$ \\
\hline Median (range) & $130.0(100.0-188.0)$ & $136.0(109.0-180.0)$ \\
\hline Missing, $n(\%)$ & $9(13.8)$ & $6(9.2)$ \\
\hline \multicolumn{3}{|l|}{ Diastolic blood pressure (mmHg) } \\
\hline Mean (SD) & $85.8(10.1)$ & $85.5(9.7)$ \\
\hline Median (range) & $86.0(66.0-108.0)$ & $84.0(68.0-107.0)$ \\
\hline Missing, $n(\%)$ & $9(13.8)$ & $6(9.2)$ \\
\hline \multicolumn{3}{|l|}{ Heart rate (bpm) } \\
\hline Mean (SD) & $74.8(10.0)$ & $72.4(10.3)$ \\
\hline Median (range) & $73.0(60.0-100.0)$ & $73.0(54.0-100.0)$ \\
\hline Missing, $n(\%)$ & $8(12.3)$ & $6(9.2)$ \\
\hline \multicolumn{3}{|l|}{ DHI total score ${ }^{a}$} \\
\hline Mean (SD) & $1.7(0.8)$ & $1.6(0.7)$ \\
\hline Median (range) & $1.7(0.4-3.5)$ & $1.5(0.4-3.1)$ \\
\hline Missing, $n(\%)$ & $1(1.5)$ & $2(3.1)$ \\
\hline \multicolumn{3}{|l|}{ Physical examination } \\
\hline \multicolumn{3}{|c|}{ Cranial nerves: head-shaking nystagmus } \\
\hline Patients with nystagmus, $n$ (\%) & $4(6.2)$ & $4(6.2)$ \\
\hline Missing, $n(\%)$ & $5(7.7)$ & $6(9.2)$ \\
\hline \multicolumn{3}{|l|}{ Coordination: Romberg's test } \\
\hline Patients with instability, $n$ (\%) & $3(4.6)$ & $4(6.2)$ \\
\hline Missing, $n(\%)$ & $2(3.1)$ & $0(0.0)$ \\
\hline \multicolumn{3}{|l|}{ Neuro-orthoptic examinations } \\
\hline \multicolumn{3}{|l|}{ Smooth pursuit eye movement } \\
\hline Saccaded, $n(\%)$ & $25(41.5)$ & $29(44.6)$ \\
\hline Missing, $n(\%)$ & $2(1.5)$ & $0(0.0)$ \\
\hline \multicolumn{3}{|l|}{ Absolute SW deviation $\left(^{\circ}\right)$} \\
\hline Mean (SD) & $0.5(1.2)$ & $0.4(1.2)$ \\
\hline
\end{tabular}


Table 1 Baseline and disease characteristics of the intention-to-treat sample (Continued)

\begin{tabular}{|c|c|c|}
\hline Characteristics & $\begin{array}{l}\text { Placebo } \\
(n=65)\end{array}$ & $\begin{array}{l}\text { Metoprolol } \\
(n=65)\end{array}$ \\
\hline Median (range) & $0.0(0.0-5.0)$ & $0.0(0.0-6.0)$ \\
\hline Missing, $n(\%)$ & $3(4.6)$ & $0(0.0)$ \\
\hline \multicolumn{3}{|c|}{ Gaze-evoked nystagmus } \\
\hline$n(\%)$ & $7(10.8)$ & $14(21.5)$ \\
\hline Missing, $n(\%)$ & $2(3.1)$ & $0(0.0)$ \\
\hline \multicolumn{3}{|c|}{ Nystagmus in the scanning laser ophthalmoscope } \\
\hline$n(\%)$ & $8(12.3)$ & $3(4.6)$ \\
\hline Missing, $n(\%)$ & $12(18.5)$ & $9(13.8)$ \\
\hline \multicolumn{3}{|c|}{ Disturbed fixation suppression } \\
\hline$n(\%)$ & $6(9.2)$ & $3(4.6)$ \\
\hline Missing, $n(\%)$ & $4(6.2)$ & $1(1.5)$ \\
\hline \multicolumn{3}{|l|}{ Oculography } \\
\hline \multicolumn{3}{|c|}{ Spontaneous nystagmus ( $\%$ s) } \\
\hline Velocity = $0, n(\%)$ & $47(72.3)$ & $48(73.8)$ \\
\hline Velocity $\geq 1, n(\%)$ & $16(24.5)$ & $16(24.5)$ \\
\hline Velocity $\geq 3, n(\%)$ & $1(1.5)$ & $1(1.5)$ \\
\hline Missing, $n(\%)$ & $2(3.1)$ & $1(1.5)$ \\
\hline \multicolumn{3}{|c|}{ Gaze-evoked nystagmus (\%/s) } \\
\hline Velocity = $0, n(\%)$ & $14(21.5)$ & $7(10.8)$ \\
\hline Velocity $\geq 1, n(\%)$ & $43(66.1)$ & $49(75.3)$ \\
\hline Velocity $\geq 3, n(\%)$ & $6(9.2)$ & $10(15.3)$ \\
\hline Missing, $n(\%)$ & $8(12.3)$ & $9(13.8)$ \\
\hline \multicolumn{3}{|c|}{ Bithermal caloric testing (normalized right-left difference (\%) according to Jongkees' formulab) } \\
\hline Mean (SD) & $-8.5(20.5)$ & $-6.9(21.9)$ \\
\hline Median (range) & $-10.8(-58.3$ to 73.0$)$ & $-5.9(-82.9$ to 71.4$)$ \\
\hline Missing, $n(\%)$ & $6(9.2)$ & $5(7.7)$ \\
\hline
\end{tabular}

SD standard deviation, SVV subjective visual vertical, VM vestibular migraine

${ }^{a}$ Dizziness handicap inventory (DHI); high score indicates high impairment; range of possible total scores, 0-100; mean total score (range 0-4) indicates averaging for the number of answered questions (see Additional file 4)

${ }^{\mathrm{b}}$ Jongkees' formula $=100 \cdot(|\mathrm{RC}|+|\mathrm{RW}|-(|\mathrm{LC}|+|\mathrm{LW}|)) /(|\mathrm{RC}|+|\mathrm{LC}|+|\mathrm{RW}|+|\mathrm{LW}|) \frac{(|\mathrm{RC}|+|\mathrm{RW}|-(|\mathrm{LC}|+|\mathrm{LW}|))}{(|\mathrm{RC}|+|\mathrm{LC}|+|\mathrm{RW}|+|\mathrm{LW}|)}$; where $\mathrm{RW}=$ right warm rinse $\left(44^{\circ} \mathrm{C}\right.$ water $), \mathrm{LW}=\mathrm{left}$ warm

rinse, $\mathrm{RC}=$ right cold rinse, $\mathrm{LC}=$ left cold rinse; the condition "warm" means irrigation with $44^{\circ} \mathrm{C}$ water; "cold" means irrigation with $30^{\circ} \mathrm{C}$ water

Hence, there were no protocol-defined adverse events of special interest. No deaths or suspected unexpected serious adverse reactions occurred during the trial. Eighteen patients (nine in the placebo group and nine in the metoprolol group) reported a total of 21 SAEs over the whole 9-month study period.

Within the maximum treatment duration of 6 months, a total of 348 AEs occurred (174 in each group) for the safety population; $18.6 \%(11 / 59)$ of patients receiving placebo were not affected by AEs compared to $16.1 \%$ (10/62) receiving metoprolol. With respect to AE severity, the incidence was similar for both groups (AEs in the placebo group: $45.1 \%$ mild, $16.2 \%$ severe; in the metoprolol group: $42.2 \%$ mild, $14.5 \%$ severe). In both groups at least three AEs occurred for $50 \%$ of patients (placebo: $49.2 \%$ (29/59); metoprolol: $50.0 \%$ $(31 / 62)$ ). Fifteen patients (nine in the placebo group and six in the metoprolol group) were affected by a total of 17 SAEs while on study treatment. Two severe, treatment-emergent SAEs (one in each group: hospitalization due to diverticulitis in the placebo group and hospitalization due to migraine in the metoprolol group; both recovered) were suspected by the investigator to be causally related to treatment. One patient receiving metoprolol discontinued the treatment owing to an SAE, and seven discontinued because of non-serious AEs, as compared to two patients on placebo because of SAEs and two because of non-serious AEs. Detailed information of the frequency of AEs which occurred within the 6 months of intervention is displayed in Table 4.

\section{Discussion}

VM is considered the most common neurologic cause of recurrent spontaneous vertigo episodes [32]. The main 
Table 2 Summary of diary-based primary and secondary end points for the full analysis set population

\begin{tabular}{|c|c|c|c|}
\hline & $N^{a}$ & Placebo & Metoprolol \\
\hline \multicolumn{4}{|l|}{ Primary end point } \\
\hline Vertigo attacks, monthly ${ }^{\mathrm{b}}$ incidence rates $(95 \% \mathrm{Cl})^{\mathrm{a}}$ & 114 & & \\
\hline Month 4 & & $4.499(3.295-5.704)$ & $4.202(3.138-5.267)$ \\
\hline Month 5 & & $3.733(2.527-4.939)$ & $3.428(2.384-4.471)$ \\
\hline Month 6 & & $3.097(1.914-4.281)$ & $2.796(1.792-3.800)$ \\
\hline Decay rate $(95 \% \mathrm{Cl}), P$ value & & \multicolumn{2}{|c|}{$0.830(0.776-0.887),<0.001$} \\
\hline IRR $(95 \% \mathrm{Cl}), P$ value & & \multicolumn{2}{|c|}{$0.983(0.902-1.071), 0.696$} \\
\hline \multicolumn{4}{|l|}{ Secondary end points } \\
\hline Vertigo days, monthly incidence rates $(95 \% \mathrm{Cl})^{\mathrm{a}}$ & 114 & & \\
\hline Month 4 & & $6.757(5.067-8.447)$ & $5.278(3.999-6.557)$ \\
\hline Month 5 & & $5.881(4.126-7.637)$ & $4.319(3.070-5.569)$ \\
\hline Month 6 & & $5.119(3.326-6.912)$ & $3.534(2.334-4.735)$ \\
\hline Decay rate $(95 \% \mathrm{Cl}), P$ value & & \multicolumn{2}{|c|}{$0.870(0.821-0.923),<0.001$} \\
\hline IRR $(95 \% \mathrm{CI}), P$ value & & \multicolumn{2}{|c|}{$0.940(0.869-1.017), 0.125$} \\
\hline Mean monthly ${ }^{\mathrm{b}} \mathrm{MHDs}^{\mathrm{c}}$ & 91 & & \\
\hline Months 4-6 & & $2.400(1.410-4.410)$ & $2.505(1.488-4.215)$ \\
\hline IRR $(95 \% \mathrm{CI}), P$ value & & \multicolumn{2}{|c|}{$1.048(0.482-2.250), 0.904$} \\
\hline $\begin{array}{l}\mathrm{Cl} \text { confidence interval } \\
\text { a Primary efficacy analysis by a Poisson generalized linear } \mathrm{m} \\
\text { assumption is maximal effect of intervention during the pre } \\
\text { supplementary analysis } \\
\text { b Mean incidence rates per } 30 \text { days derived by a model-bas } \\
\text { 6; reference group }=\text { placebo } \\
\text { c Migraine headache days (MHDs): rates and incidence rate } \\
\text { aggregated MHD data reported within months } 4-6 \text { only ( } 91\end{array}$ & ming & $\begin{array}{l}\text { lope) based on the wh } \\
\text { onths 4-6; analysis of } v \\
\text { dom; pre-specified tim } \\
\text { e binomial generalized } \\
\text { documentation) }\end{array}$ & $\begin{array}{l}\text { ment period; } \\
\text { oerformed as a } \\
\text { ry interest months 4- } \\
\text { ed on the }\end{array}$ \\
\hline
\end{tabular}

reason for the diagnostic challenge is the broad spectrum of its manifestations, e.g. episodic vestibular symptoms without typical migraine headaches, and the wide variety of ictal and interictal symptoms [33].

\section{Principal findings}

The PROVEMIG trial proved not to be feasible regarding patient recruitment and was terminated early after randomization of 130 patients, i.e. after achieving about $50 \%$ of the target enrolment. Nevertheless, there are several important findings and lessons that can be learnt from our study to inform future interventional drug trials in this patient population and to apply methods of quantitative evidence synthesis in terms of meta-analyses.

The key finding of the trial are as follows. First, in both randomized groups, patients experienced a significant reduction in the monthly incidence of vertigo attacks (according to the definition used in this study, i.e. lasting between $5 \mathrm{~min}$ and no longer than $72 \mathrm{~h}$ ) of $17.0 \%$ (95\% CI 11.3-22.4\%) over the whole double-blind 6-month treatment period. However, prophylactic treatment with the beta blocker metoprolol was not superior to placebo in diminishing the monthly incidence of vertigo attacks over time (IRR 0.983 (95\% CI 0.902-1.071)). Although the trial was prematurely ended, the 95\% CIs for the estimated decay rate and IRR are rather narrow; apparently, large treatment effects are not very likely given the present results. Second, no beneficial therapeutic effect of metoprolol could be established either in the patient-reported efficacy outcomes (including the DHI total score measured by a psychometrically validated questionnaire) or in the clinical assessments. Third, the investigational drug and placebo regimens were approximately equally safe and well tolerated in the participating patients, with no unexpected safety findings.

In summary, there is no evidence from randomized controlled trials to support or refute treatment with metoprolol in patients diagnosed with VM.

\section{Comparison with previous literature}

To our knowledge, this is the first report of a pragmatic, double-blind, randomized, placebo-controlled trial investigating the effectiveness, tolerability and safety of a prophylactic symptomatic treatment with metoprolol compared to placebo in patients with VM. The first Cochrane systematic review on this topic published in 2015 aimed to assess the effects of pharmacological agents (including beta blockers) used in the prophylactic treatment of VM-associated symptoms against placebo 

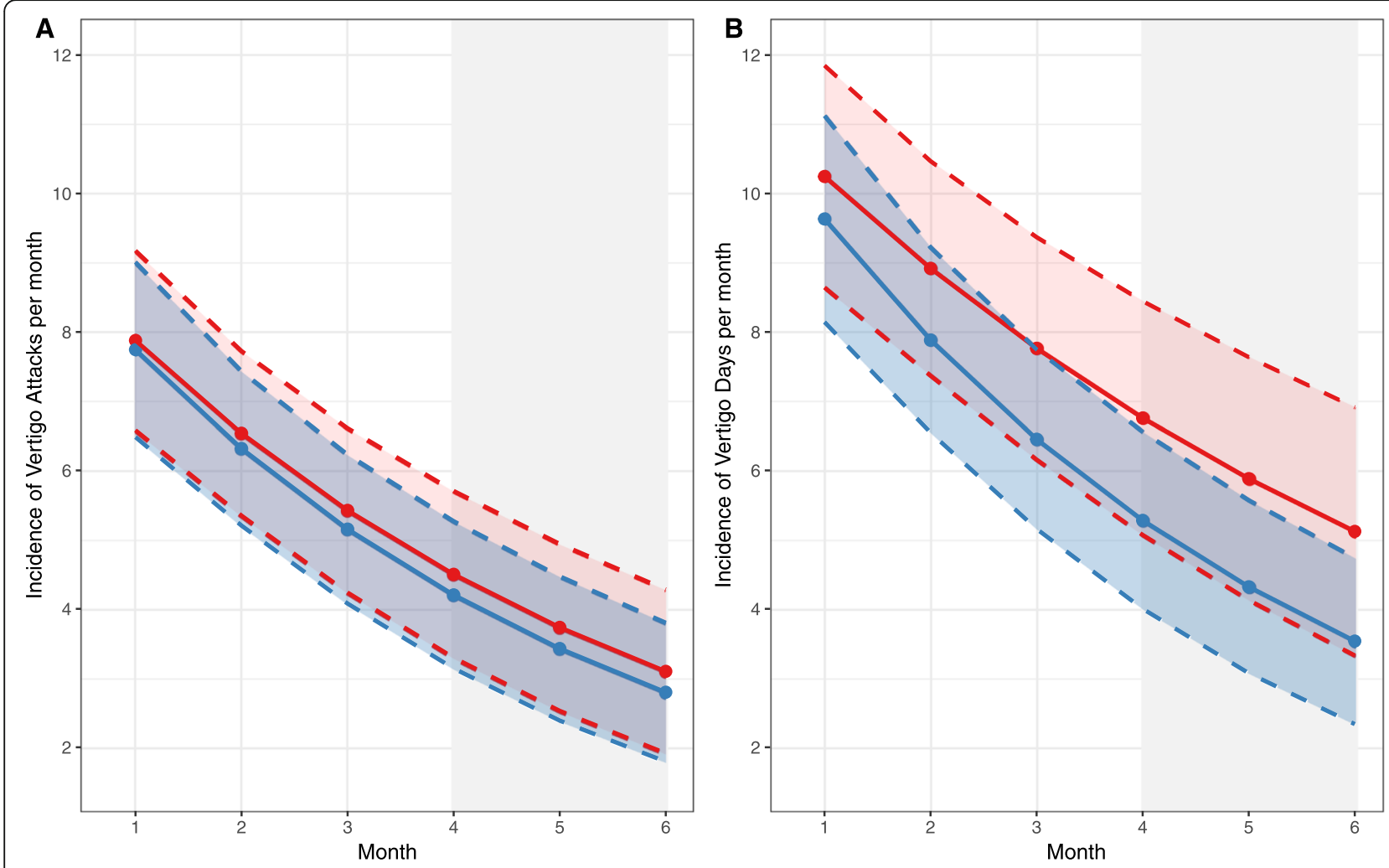

Treatment
group

Fig. 2 Predicted marginal means (with pointwise 95\% confidence intervals) for (a) the incidence of vertigo attacks (primary efficacy outcome) and (b) vertigo days per 30 days during the 6-month treatment period (full analysis set population). A Poisson random intercept and slope model was applied for the principal analysis. The grey shaded area represents the 90-day time period of primary interest comprising of estimated monthly incidences rates for months 4 to 6

or no treatment ("wait-and-see"). However, the authors did not find any evidence from completed randomized controlled trials using the Bárány Society/International Headache Society diagnostic criteria, while identifying PROVEMIG as the only ongoing trial fulfilling the inclusion criteria with respect to trial design [9].

\section{Strengths and weaknesses}

The trial population consisted of 130 patients (with 121 patients commencing the allocated intervention) selected from 527 patients screened for eligibility. Patients were diagnosed according to the established Neuhauser criteria 2001 [2]. The current International Classification of Vestibular Disorders criteria for VM were not available at that time, which are mostly similar to "definite VM" according to Neuhauser. Investigators at the participating trial sites were clinical experts in the diagnosis and treatment of vestibular disorders. Due to the complexity of the disease entity, expert knowledge with respect to diagnosis is essential in order to differentiate between VM and other diseases with spontaneous recurrent vertigo, most notably Menière's disease [34-36]. Baseline assessments (Table 1) provide neuro-otological and ophthalmological data systematically collected from a well-defined sample of VM patients. The female preponderance in the study population was found to be 1.5:1 which is consistent with the 1.5-5:1 female-to-male ratio reported in other studies [2, 37].

The 6-month duration of treatment allowed us to ascertain whether the active agent may be distinguished from placebo by how quickly patients achieve a reduction in monthly vertigo attacks ('speed of effect'). Since the target was comparing the benefit of two treatment policies (i.e. the drug as actually taken), all patients were allowed to take rescue medication if necessary. About $68 \%$ of patients comprising the per-protocol population were at least 3 months on treatment. Altogether, the proportion of missing data with respect to the diary-based efficacy outcomes was not higher than expected for symptomatic trials assessing the ability of an intervention to provide symptom relief from the condition. 
Table 3 Key secondary outcome results (least square mean change difference or odds ratio) ${ }^{a}$

\begin{tabular}{|c|c|c|c|}
\hline Secondary End points & $N^{b}$ & Placebo & Metoprolol \\
\hline $\mathrm{DHI}$ mean total score & 91 & & \\
\hline LS mean change ${ }^{c}(95 \% \mathrm{Cl})$ & & $0.159(-0.252$ to 0.570$)$ & $0.080(-0.310$ to 0.470$)$ \\
\hline Difference vs. placebo (95\% Cl) & & \multicolumn{2}{|c|}{$-0.079(-0.360$ to 0.201$)$} \\
\hline$P$ value & & \multicolumn{2}{|c|}{0.577} \\
\hline Pursuit eye movement & 92 & & \\
\hline Patients achieving response ${ }^{d}, n(\%)$ & & $5(11.6)$ & $8(16.3)$ \\
\hline OR $(95 \% \mathrm{Cl})$ & & $0.132(0.045-0.305)$ & $0.195(0.092-0.416)$ \\
\hline Difference vs. placebo, OR (95\% Cl) & & \multicolumn{2}{|c|}{$1.483(0.454-5.277)$} \\
\hline$P$ value & & \multicolumn{2}{|c|}{0.520} \\
\hline SW & 90 & & \\
\hline Patients achieving response ${ }^{d}, n(\%)$ & & $4(9.5)$ & $2(4.2)$ \\
\hline OR $(95 \% \mathrm{Cl})$ & & $0.105(0.032-0.262)$ & $0.043(0.012-0.179)$ \\
\hline Difference vs. placebo, OR (95\% Cl) & & \multicolumn{2}{|c|}{$0.413(0.055-2.235)$} \\
\hline$P$ value & & \multicolumn{2}{|c|}{0.322} \\
\hline $\begin{array}{l}\text { Cl confidence interval } \\
\text { a Least square (LS) mean change differenc } \\
\text { derived by logistic regression (unadjusted) } \\
\text { covariance; for change state from baseline } \\
\text { (FAS) sample } \\
\text { b Numbers of patients with non-missing o } \\
\text { c Change score means difference between } \\
\text { d Logistic regression for the change state } \\
\text { otherwise); pursuit eye movement - treat }\end{array}$ & baseli & $\begin{array}{l}\text { of covariance for absolute } \\
\text { month } 6 \text { for Dizziness Han } \\
\text { isual vertical (SVV) by logis } \\
\text { (FAS population: } n=127 \text {; } \\
\text { eline score; see Table } 1 \text { for } \\
\text { en baseline and month } 6 \text { ( } \\
0 \text { 'smooth' }\end{array}$ & $\begin{array}{l}\text { ds ratio (OR; estimates } \\
\text { by analysis of } \\
\text { full analysis set } \\
\text { orolol } n=64) \\
\text { ore ranges } \\
\text { rmal to normal; } 0 \text {, }\end{array}$ \\
\hline
\end{tabular}

In this trial, the experimental treatment with the beta blocker metoprolol was compared in a blinded manner with an unspecific treatment, i.e. placebo, which is assumed to cover all the unspecific effects of an intervention (e.g. patient expectations, natural course or regression-to-the-mean) [38]. The findings for patients receiving placebo may not fully reflect the natural course of VM which has been reported to vary over time.

Our study has certain limitations. Faced with persisting recruitment difficulties, the trial was not successful in reaching the target sample size. Therefore, we cannot perform confirmatory analyses, but provide 95\% CIs for estimators of treatment effectiveness. One factor that led to delays and early trial closure was the number of screening failures being higher than expected. Reasons for the poor participant accrual were multifactorial and included unwillingness to accept the underlying intervention and failure to meet eligibility criteria (in particular, a low baseline frequency with respect to attacks of VM, or comorbidities being contraindications for metoprolol). Furthermore, the number of participating trial sites was lower than anticipated. With $84 \%$ of 130 randomized patients recruited at a single site, the highly specialized dizziness unit at the Ludwig Maximilians University, Munich, which attracts patients from all over Germany, our

Table 4 Safety assessment by study treatment group (safety sample) during the 6-month treatment period

\begin{tabular}{lll}
\hline Safety assessment & $\begin{array}{l}\text { Placebo } \\
(n=59)\end{array}$ & $\begin{array}{c}\text { Metoprolol } \\
(n=62)\end{array}$ \\
\hline Deaths, $n$ & 0 & 0 \\
Patients with SUSARs, $n$ & 0 & 0 \\
Patients with early termination from the study due to SAEs ${ }^{\mathrm{a}}, n(\%)$ & $2(3.4)$ & $1(1.6)$ \\
Treatment-related SAEs, $n$ (\%) & $8(1.7)$ & $1(1.6)$ \\
Patients with at least one SAE, $n$ (\%); total number of SAEs & $4(9.7) ; 10$ & $8(12.9)$ \\
\hline Patients with early termination due to adverse events ${ }^{\mathrm{a}}, n(\%)$ & $4(6.7)$ & \\
\hline
\end{tabular}

Percentages (\%) are based on the number of patients in the safety sample

Reasonable possibility for a causal relationship = drug-event relationship reported as "possible", "probable", or missing according to the adverse event case report form

${ }^{a}$ Adverse event or serious adverse event (SAE) leading to treatment discontinuation according to the adverse event case report form

SUSAR suspected unexpected serious adverse reactions 
findings may not be applicable to all German patients with VM. Due to the considerable overlap of the two disorders of VM and Menière's disease, the study population might be contaminated.

Since VM is a complex and relatively new single disease entity, clinically meaningful patient-centred primary efficacy outcomes are still being debated. In this trial, a patient dizziness diary routinely used in clinical practice for diagnosis at the site of the principal investigator was adapted for the clinical trial setting. Paperbased daily diaries are prone to errors or being lost and have no simple methods for backup and reconstruction of information. If paper-based symptom diaries are applied for tracking recurrent events and to understand longitudinal relationships over time in confirmatory clinical trials, patient recordings have to be reviewed by the site personnel for accuracy and interpretability of reported symptoms in order to derive rigidly defined primary efficacy end points. To face this dilemma, further research is warranted to define responsive patientcentred outcomes for both outcome domains (vertigo and migraine headache) that have been shown to be important to patients and clinicians to allow informed decision making. Since patients with VM experience a wide variety of ictal and interictal symptoms, more effort should be made in investigating whether established outcome measurement instruments for both domains are appropriate for this target population, and in evaluating the quality of these instruments (primarily with respect to responsiveness to change) [39]. As such, the validated scores derived from the self-reported DHI questionnaire together with self-administered migraine-specific qualityof-life questionnaires such as the Migraine Disability Assessment or the Headache Impact Test might be used to evaluate the treatment benefit while reducing the documentation burden for the patient compared to a diarybased measurement of disability which could be essential for long-term comparative effectiveness trials [40-44].

The following points should be mentioned with respect to the PROVEMIG diary (Additional file 3):

1) With headache as a co-primary end point, migraine headache symptoms should have been assessed in a dedicated column on the diary form instead of one of the potential accompanying symptoms for vertigo episodes.

2) A simple visual analogue scale integrated in the diary might have helped patients, leading to a better rating with respect to the severity of symptoms (primarily with a lower rate of missing data).

3) Daily diary entries have to be regularly monitored by the site personnel during study visits (in order to distinguish the absence of episodes from noncompliance with diary maintenance). In the case of electronic diaries which could be used in future trials (ideally based on an app which can remind the patient on a daily basis to update) this can be continuously done online and assisted by algorithms, which potentially would improve data quality provided that they give useful guidance rather than bossing around the patient leaving them frustrated.

4) Regardless if a paper-based or e-diary is applied, scoring algorithms to retrieve the pre-specified efficacy end points have to be established and validated. Without doing so, we would not have been able to carry out meaningful longitudinal analyses of the PROVEMIG diary data.

\section{Conclusions}

It is of the utmost importance to develop a core outcome set for this complex vestibular disease comprising of symptoms attributable to both vertigo and migraine headache, with the aim of reducing the documentation burden for the trial participants in long-term trials and defining clinically meaningful, patient-centred efficacy end points that are sensitive to change and that are reproducible [45-47]. A strong placebo response was observed in the PROVEMIG trial which is well known from other trials of migraine treatment [48].

For future phase III trials, a more efficient site set-up and improved recruitment methods would seem to be appropriate. Furthermore, study site personnel should aim to follow patient retention strategies to ensure that participants, once recruited, are engaged clinically and followed up as completely as possible to avoid noncompliance with diary maintenance in particular. Additional multicentre, randomized, placebo-controlled trials are needed to replicate these findings and to explore subgroups of patients reporting response to antihypertensive drugs.

\section{Supplementary information}

Supplementary information accompanies this paper at https://doi.org/10. 1186/s13063-019-3903-5.

Additional file 1. Consolidated Standards of Reporting Trials (CONSORT) 2010 checklist of information to include when reporting a randomized trial.

Additional file 2. Original study protocol.

Additional file 3. Patient symptom diary (original German version; word-for-word English translation).

Additional file 4. Procedural and statistical methods.

Abbreviations

AE: Adverse event; BPPV: Benign paroxysmal positional vertigo; Cl: Confidence interval; DHI: Dizziness Handicap Inventory; FAS: Full analysis set; IRR: Incidence rate ratio; MAR: Missing at random; MHD: Migraine headache day; OR: Odds ratio; SAE: Serious adverse event; SW: Subjective visual vertical; VM: Vestibular migraine 


\section{Acknowledgments}

The authors thank all patients enrolled in the present study and their families, the study centres, and investigators who took part in this trial. The authors would like to thank Hans-Helge Müller for substantially contributing to the conception and design including the statistical planning, and for his valuable advice and suggestions. The authors are grateful to Ivonne Naumann at the site of the co-ordinating investigator for providing the screening information for the non-eligible patients, and to Josef Herker at the IBE, LMU Munich, for data management.

This article is published on behalf of the PROVEMIG investigators (Additional file 4): Michael Strupp (Hospital of the University of Munich, Clinic for Neurology and German Center for Vertigo and Balance Disorders, Munich, Germany), Hans-Christoph Diener (Hospital of the University of Essen, Department of Neurology, Essen, Germany), Hubert Löwenheim (Hospital of the University of Tübingen, Department of ENT, Tübingen, Germany), Thomas Lempert (Schlosspark-Klinik, Department of Neurology, Berlin, Germany), Wolfgang Heide (Allgemeines Krankenhaus, Neurology, Celle, Germany), Holger A Rambold (Kreiskliniken Altötting-Burghausen, Neurologische Klinik, Altötting, Germany).

\section{Authors' contributions}

$\mathrm{OB}$ and $\mathrm{CA}$ have shared first authorship. MS, OB, CA and UM contributed to the design of the trial, wrote the study protocol or preceding research proposals leading to the funding of the trial, and interpreted the work. As coordinating investigator, MS initiated the collaborative clinical trial project and is the guarantor. MS, OB, and all investigators of the recruiting study sites acquired the data. OB developed the rules to derive efficacy outcomes based on the raw daily diary recordings and is responsible for the corresponding SAS algorithm. AAT wrote the statistical analysis plan; AAT and CA performed the statistical analyses. AAT, CA and UM interpreted the results. $\mathrm{CA}$ and $\mathrm{OB}$ wrote the first draft and all subsequent versions of the manuscript. All authors revised the work critically for important intellectual content and agree to be accountable for all aspects of the work in ensuring that questions related to the accuracy or integrity of any part of the work are appropriately investigated and resolved. The corresponding author attests that all listed authors meet authorship criteria and that no others meeting the criteria have been omitted. All authors read and approved the final manuscript.

\section{Authors' information}

MS is professor of Neurology at the Department of Neurology and the German Center for Vertigo and Balance Disorders (DSGZ) at the LMU Munich. UM is director and chair of biostatistics and bioinformatics at the Institute for Medical Information Processing, Biometry, and Epidemiology (IBE) at the LMU Munich. CA is a biostatistician and postdoctoral researcher at the IBE, LMU Munich. AAT is a pharmacist and holds a Master of Science degree in Epidemiology from the LMU Munich. OB is a medical doctor and, as head of the clinical study center at the DSGZ, LMU Munich, he was the project manager of the PROVEMIG trial.

\section{Funding}

The PROVEMIG study was not co-sponsored and was supported by the German Center for Vertigo and Balance Disorders (DSGZ), University Hospital Munich, Campus Grosshaden, funded by the German Federal Ministry of Education and Research within the framework of the Integrated Research and Treatment Centers program (funding reference number: 01EO0901). The funder had no role in the design, management, data collection, analyses, or interpretation of the data or in writing the manuscript or the decision to submit for publication.

\section{Availability of data and materials}

Data cannot be shared publicly because participants did not explicitly consent to the sharing of their data as per the European Union's General Data Protection Regulation and the corresponding German privacy laws. Data are available through the Research Ethics Board of the LudwigMaximilians-Universität, Munich, Germany, for researchers who meet the criteria for access to confidential data. Please address requests to ethikkommission@med.uni-muenchen.de (ethics committee of the LMU Munich) and to the biostatistician at mansmann@ibe.med.uni-muenchen.de.

\section{Ethics approval and consent to participate}

Written informed consent was obtained from all participants before initiation of the first study-specific procedure. The study protocol, including the patient information and consent form, was approved by the local ethics committee of each participating institution (leading ethics committee: ethics committee of the Medical Faculty of the Ludwig-Maximilians-Universität, Munich, Germany; reference number: 152-11 fed) and by Germany's Federal Institute for Drugs and Medical Devices (BfArM; project number 4037229). Clinical trial authorisation was granted on 1 September 2011 (sponsor's protocol code number: VMMET009). The trial was performed in accordance with the Declaration of Helsinki and other applicable guidelines, laws, and regulations.

\section{Consent for publication}

Consent forms for the trial included consent for publication of results in peer-reviewed journals.

\section{Competing interests}

All authors have completed the ICMJE uniform disclosure form at www. icmje.org/coi_disclosure.pdf (available on request from the corresponding author). MS is Joint Chief Editor of the Journal of Neurology, Editor in Chief of Frontiers of Neuro-otology and Section Editor of F1000. He has received speaker's honoraria from Abbott, Actelion, Auris Medical, Biogen, Eisai, Grünenthal, GSK, Henning Pharma, Interacoustics, MSD Sharp \& Dohme, Otometrics, Pierre-Fabre, TEVA GmBH, and UCB. He is a shareholder in IntraBio. He acts as a consultant for Abbott, Actelion, AurisMedical, Heel, IntraBio and Sensorion. The remaining authors declare that they have no competing interests.

\section{Author details}

${ }^{1}$ German Center for Vertigo and Balance Disorders (DSGZ), Ludwig Maximilians University, Campus Grosshadern, Munich, Germany. ${ }^{2}$ ReliaTec $\mathrm{GmbH}$, Garching, Germany. ${ }^{3}$ Institute for Medical Information Processing, Biometry and Epidemiology (IBE), Ludwig Maximilians University, Campus Grosshadern, Marchioninistr. 15, 81377 Munich, Germany. ${ }^{4}$ Department of Neurology, Ludwig Maximilians University, University Hospital Munich, Campus Grosshadern, Munich, Germany.

Received: 20 March 2019 Accepted: 12 November 2019 Published online: 30 December 2019

\section{References}

1. Headache Classification Committee of the International Headache Society (IHS). The international classification of headache disorders, 3rd edition. Cephalalgia. 2018;38(1):1-211.

2. Neuhauser $H$, Leopold M, von Brevern M, Arnold G, Lempert T. The interrelations of migraine, vertigo, and migrainous vertigo. Neurology. 2001; 56(4):436-41.

3. Lempert T, Olesen J, Furman J, Waterston J, Seemungal B, Carey J, Bisdorff A, Versino M, Evers S, Newman-Toker D. Vestibular migraine: diagnostic criteria. J Vestib Res. 2012;22(4):167-72.

4. Furman JM, Marcus DA, Balaban CD. Migrainous vertigo: development of a pathogenetic model and structured diagnostic interview. Curr Opin Neurol. 2003;16(1):5-13.

5. Marcus DA, Kapelewski C, Rudy TE, Jacob RG, Furman JM. Diagnosis of migrainous vertigo: validity of a structured interview. Med Sci Monit. 2004; 10(5):CR197-201.

6. Neuhauser HK. Epidemiology of vertigo. Curr Opin Neurol. 2007;20(1):40-6.

7. Formeister EJ, Rizk HG, Kohn MA, Sharon JD. The epidemiology of vestibular migraine: a population-based survey study. Otol Neurotol. 2018;39(8):1037-44.

8. Strupp M, Versino M, Brandt T. Vestibular migraine (Chapter 62). Handbook of Clinical Neurology. In: Aminoff MJ, Boller F, Swaab DF, editors. Headache, Vol. 97. Amsterdam: Elsevier; 2010. p. 755-71. https://doi.org/10.1016/S00729752(10)97062-0.

9. Maldonado Fernandez M, Birdi JS, Irving GJ, Murdin L, Kivekas I, Strupp M. Pharmacological agents for the prevention of vestibular migraine. Cochrane Database Syst Rev. 2015;(6):Cd010600.

10. Lauritsen CG, Marmura MJ. Current treatment options: vestibular migraine. Curr Treat Options Neurol. 2017;19(11):38.

11. Stolte B, Holle D, Naegel S, Diener HC, Obermann M. Vestibular migraine. Cephalalgia. 2015;35(3):262-70. 
12. Bisdorff AR. Treatment of migraine related vertigo with lamotrigine an observational study. Bull Soc Sci Med Grand Duche Luxemb. 2004;2:103-8.

13. Carmona S, Settecase N. Use of topiramate (topamax) in a subgroup of migraine-vertigo patients with auditory symptoms. Ann N Y Acad Sci. 2005; 1039(1):517-20.

14. Reploeg MD, Goebel JA. Migraine-associated dizziness: patient characteristics and management options. Otol Neurotol. 2002;23(3):364-71.

15. Lepcha A, Amalanathan S, Augustine AM, Tyagi AK, Balraj A. Flunarizine in the prophylaxis of migrainous vertigo: a randomized controlled trial. Eur Arch Otorhinolaryngol. 2014;271(11):2931-6.

16. Salviz M, Yuce T, Acar H, Karatas A, Acikalin RM. Propranolol and venlafaxine for vestibular migraine prophylaxis: a randomized controlled trial. Laryngoscope. 2016;126(1):169-74.

17. Evers S, Afra J, Frese A, Goadsby PJ, Linde M, May A, Sandor PS. European Federation of Neurological S: EFNS guideline on the drug treatment of migraine-revised report of an EFNS task force. Eur J Neurol. 2009;16(9): 968-81.

18. Diener HC, Katsarava Z, Limmroth V. Current diagnosis and treatment of migraine. Ophthalmologe. 2008;105(5):501-8 quiz 509-510.

19. Moher D, Hopewell S, Schulz KF, Montori V, Gøtzsche PC, Devereaux PJ, Elbourne D, Egger M, Altman DG. CONSORT 2010 explanation and elaboration: updated guidelines for reporting parallel group randomised trials. BMJ. 2010;340:c869.

20. Calvert M, Blazeby J, Altman DG, Revicki DA, Moher D, Brundage MD, Group CP. Reporting of patient-reported outcomes in randomized trials: the CONSORT PRO extension. JAMA. 2013;309(8):814-22.

21. Butcher NJ, Monsour A, Mew EJ, Szatmari P, Pierro A, Kelly LE, Farid-Kapadia M, Chee-a-tow A, Saeed L, Monga S, et al. Improving outcome reporting in clinical trial reports and protocols: study protocol for the Instrument for reporting Planned Endpoints in Clinical Trials (InsPECT). Trials. 2019;20(1):161.

22. Jacobson GP, Newman CW. The development of the Dizziness Handicap Inventory. Arch Otolaryngol Head Neck Surg. 1990;116(4):424-7.

23. Kurre A, van Gool CJ, Bastiaenen CH, Gloor-Juzi T, Straumann D, de Bruin ED. Translation, cross-cultural adaptation and reliability of the German version of the dizziness handicap inventory. Otol Neurotol. 2009:30(3):359-67.

24. CHMP. ICH E9 (R1) Addendum on estimands and sensitivity analysis in clinical trials to the guideline on statistical principles for clinical trials. EMA/ CHMP/ICH/436221/201, Step 4 (Final Version adopted on 20 Nov 2019). European Medicines Agency, Committee for Medicinal Products for Human Use (CHMP); 2019. https://database.ich.org/sites/default/files/E9-R1_Step4_ Guideline_2019_1203.pdf. Accessed 17 Dec 2019.

25. Molenberghs G, Thijs H, Jansen I, Beunckens C, Kenward MG, Mallinckrodt C Carroll RJ. Analyzing incomplete longitudinal clinical trial data. Biostatistics. 2004:5(3):445-64

26. O'Kelly M, Ratitch B. Clinical trials with missing data: a guide for practitioners. Chichester: Wiley; 2014.

27. White IR, Carpenter J, Horton NJ. Including all individuals is not enough: lessons for intention-to-treat analysis. Clin Trials. 2012:9(4):396-407.

28. CHMP. Guideline on clinical investigation of medicinal products for the treatment of migraine. In: CPMP/EWP/788/2001 Rev 1. European Medicines Agency, Committee for Medicinal Products for Human Use (CHMP); 2007. https://www.ema.europa.eu/en/documents/scientific-guideline/quidelineclinical-investigation-medicinal-products-treatment-migraine_en.pdf. Accessed 17 Dec 2019

29. R Development Core Team. R: a language and environment for statistical computing. Vienna: R Foundation for Statistical Computing, http://www.Rproject.org/; 2019

30. Bates D, Maechler M, Bolker B, Walker S: Ime4: Linear mixed-effects models using Eigen and S4, R package version 1.1-7. http://CRAN.R-project.org/ package $=$ Ime4; 2014

31. Bates D, Maechler M, Bolker B, Walker S: Fitting linear mixed-effects models using Ime4. ArXiv e-print, http://arxiv.org/abs/1406.5823; 2014.

32. Obermann M. Editorial: vestibular migraine. Front Neurol. 2017:8:213.

33. Beh SC, Masrour S, Smith SV, Friedman DI. The spectrum of vestibular migraine: clinical features, triggers, and examination findings. Headache. 2019;59(5):727-40

34. Radtke A, Neuhauser $\mathrm{H}$, von Brevern M, Hottenrott T, Lempert T. Vestibular migraine - validity of clinical diagnostic criteria. Cephalalgia. 2011;31(8):906-13.
35. Strupp M, Lopez-Escamez JA, Kim JS, Straumann D, Jen JC, Carey J, Bisdorff A, Brandt T. Vestibular paroxysmia: diagnostic criteria. J Vestib Res. 2016; 26(5-6):409-15.

36. Lopez-Escamez JA, Dlugaiczyk J, Jacobs J, Lempert T, Teggi R, von Brevern M, Bisdorff A. Accompanying symptoms overlap during attacks in Meniere's disease and vestibular migraine. Front Neurol. 2014;5:265.

37. Lempert T. Vestibular migraine. Semin Neurol. 2013;33(03):212-8.

38. Wood L, Egger M, Gluud LL, Schulz KF, Jüni P, Altman DG, Gluud C, Martin RM, Wood AJG, Sterne JAC. Empirical evidence of bias in treatment effect estimates in controlled trials with different interventions and outcomes: meta-epidemiological study. BMJ. 2008:336(7644):601-5.

39. Prinsen CA, Vohra S, Rose MR, Boers M, Tugwell P, Clarke M, Williamson PR, Terwee CB. How to select outcome measurement instruments for outcomes included in a "Core Outcome Set" — a practical guideline. Trials. 2016;17(1):449.

40. Benz T, Lehmann S, Gantenbein AR, Sandor PS, Stewart WF, Elfering A Aeschlimann AG, Angst FJH. Translation, cross-cultural adaptation and reliability of the German version of the migraine disability assessment (MIDAS) questionnaire. 2018;16(1):42.

41. Yang M, Rendas-Baum R, Varon SF, Kosinski M. Validation of the Headache Impact Test (HIT-6) across episodic and chronic migraine. Cephalalgia. 2011; 31(3):357-67.

42. Martin M, Blaisdell B, Kwong JW, Bjorner JB. The short-form Headache Impact Test (HIT-6) was psychometrically equivalent in nine languages. J Clin Epidemiol. 2004:57(12):1271-8.

43. Park JW, Shin HE, Kim JS, Lee KS. Assessing migraine disability by diarybased measurement: relationship to the characteristics of individual headache attacks. Eur J Neurol. 2008;15(8):817-21.

44. Stewart WF, Lipton RB, Kolodner KB, Sawyer J, Lee C, Liberman JN. Validity of the Migraine Disability Assessment (MIDAS) score in comparison to a diary-based measure in a population sample of migraine sufferers. Pain. 2000;88(1):41-52.

45. Kirkham JJ, Davis K, Altman DG, Blazeby JM, Clarke M, Tunis S, Williamson PR. Core Outcome Set-STAndards for Development: the COS-STAD recommendations. PLoS Med. 2017;14(11):e1002447.

46. Smith H, Horobin A, Fackrell K, Colley V, Thacker B, Hall DA. Defining and evaluating novel procedures for involving patients in Core Outcome Set research: creating a meaningful long list of candidate outcome domains. Res Involv Engagem. 2018;4:8.

47. Williamson PR, Altman DG, Bagley H, Barnes KL, Blazeby JM, Brookes ST, Clarke M, Gargon E, Gorst S, Harman N, et al. The COMET handbook: version 1.0. Trials. 2017;18(Suppl 3):280

48. Antonaci F, Chimento P, Diener HC, Sances G, Bono G. Lessons from placebo effects in migraine treatment. J Headache Pain. 2007;8(1):63-6.

\section{Publisher's Note}

Springer Nature remains neutral with regard to jurisdictional claims in published maps and institutional affiliations.

Ready to submit your research? Choose BMC and benefit from:

- fast, convenient online submission

- thorough peer review by experienced researchers in your field

- rapid publication on acceptance

- support for research data, including large and complex data types

- gold Open Access which fosters wider collaboration and increased citations

- maximum visibility for your research: over $100 \mathrm{M}$ website views per year

At BMC, research is always in progress.

Learn more biomedcentral.com/submission 